\title{
Expression analysis of key enzymes involved in the accumulation of iridoid in Rehmannia glutinosa
}

\author{
Hongying Duan, Wenxiao Liu", Yunpeng Zeng \#, Wenjing Jia, Huihui Wang, Yanqing Zhou* \\ College of Life Sciences, Henan Normal University, Xinxiang 453007, P. R. China \\ *Corresponding author: dxdhy@126.com, Luckyqing2004@126.com \\ \#These authors contributed equally to this work
}

\begin{abstract}
As the traditional Chinese herb, Rehmannia glutinosa ( $R$. glutinosa) has significant effects on health. The main active ingredient of $R$. glutinosa is iridoid. In this study, root, stem and leaf of $R$. glutinosa at five different growth stages were collected, the content of iridoid in $R$. glutinosa was determinated, and the expression of key enzymes in $R$. glutinosa was analyzed by quantitative real-time PCR (q-PCR). We found that the content of iridoid was increased continuously during the first three growth stages (I-III) of $R$. glutinosa. It reached the maximum value in root and leaf at growth stage III of $R$. glutinosa. However, the content of iridoid was not lower in leaf, compared with root. 1-deoxy-D-xylulose-5-phosphate reductoisomerase (DXR), Geranyl pyrophosphate synthase (GPPS), Geraniol 10-hydroxylase (G1OH) and 10-hydroxygeraniol oxidoreductase (10HGO) were the key enzymes involved in the synthesis of iridoid in plant, but their expression pattern or level was different in various tissues and stages of $R$. glutinosa. All appeared tissue specificity and their expression was related to the accumulation of iridoid in $R$. glutinosa. Thus, DXR, GPPS, G10H and $10 \mathrm{HGO}$ might take part in the synthesis of iridoid in $R$. glutinosa and could be differently regulated during the growth and development of $R$. glutinosa, which would provide theoretical basis for the research on secondary metabolism of iridoid in $R$. glutinosa.
\end{abstract}

Keywords: Rehmannia glutinosa; iridoid; secondary metabolism; qRT-PCR.

Abbreviations: IPP_isopentenyl pyrophosphate; DMAPP_dimethylallyl pyrophosphate; MVP_mevalonate pathway; MEP_2C-methyl-D-erythritol-4-phosphate pathway; DXR_1-deoxy-D-xylulose-5-phosphate reductoisomerase; GPPS_Geranyl pyrophosphate synthase; $\mathrm{G10H}$ _Geraniol 10-hydroxylase; 10HGO_10-hydroxygeraniol oxidoreductase; R. glutinosa_Rehmannia glutinosa; qRT-PCR_quantitative real-time PCR; UV_ultraviolet; ANNOVA_analysis of variance.

\section{Introduction}

Terpenoid is one of the largest family of secondary metabolites in plant, and could be divided into monoterpene (C10), sesquiterpene (C15), diterpene (C20), triterpenes (C30) and polyterpenes (Ashour et al., 2010). Terpenoid is synthesized from precursors of isopentenyl pyrophosphate (IPP) and dimethylallyl pyrophosphate (DMAPP) (Newman and Chappell, 2008). There are two synthetic pathway of IPP in plant, such as mevalonate pathway (MVP) and 2C-methyl-D-erythritol-4phosphate pathway (MEP) (Laule et al., 2003). Iridoid is one kind of monoterpenes, and its synthesis may be completed by MEP pathway in the plant (Li et al., 2010).

In MEP pathway, 1-deoxy-D-xylulose-5-phosphate reductoisomerase (DXR) is the branch point of carbon flow, and could convert 1-deoxy-D-xylulose-5-phosphate into methyl erythritol phosphate, which is the important precursors in the synthesis of terpenoid (Luo et al., 2003). Geranyl pyrophosphate synthase (GPPS) is one of key enzymes in MEP pathway. It can catalyze the synthesis of C10 skelecton in monoterpene (Yu et al., 2013). For example, IPP and DMAPP are synthesized by
GPPS (Yang et al., 2016). Furthermore, Geraniol 10-hydroxylase $(\mathrm{G} 10 \mathrm{H})$ could catalyze geraniol hydroxylated into 10-hydroxy geraniol in the synthesis of iridoid (Sung et al., 2011), and would control the first committed step in the synthesis of secologanin and TIAs (Wang et al., 2010). The oxidation of 10-hydroxygeraniol to aldehyde 10-oxogeraniol is catalyzed by 10-hydroxygeraniol oxidoreductase (10HGO) (Verma et al., 2012). The dehydrogenases isolated from $C$. roseus may be responsible for this reaction and were able to produce iridoidial as incubated in the presence of iridoid-related synthase (Thamm et al., 2016).

Rehmannia glutinosa ( $R$. glutinosa) belongs to one kind of perennial herb, has a lot of functions, such as nourishing yin, supplementing kidney, blood hemostasis, and so on (Kang and kim, 2011). There are a variety of compounds in R. glutinosa, such as iridoid, sesquiterpene, phenylethanoid glycoside and triterpene (Zhang et al., 2013, Zhou et al., 2018). Iridoid is the main active ingredient of $R$. glutinosa, and more than 30 kinds of iridoids have been identified from $R$. glutinosa. However, the research on biosynthesis and metabolism regulation of 
iridoid in $R$. glutinosa is scarce. In order to explore the synthesis of iridoid in $R$. glutinosa, the accumulation of iridoid was analyzed, and the expression of some key enzymes in the synthesis of iridoid in $R$. glutinosa was studied by quantitative real-time PCR (qRT-PCR). Therefore, this research will help to study metabolic pathway of iridoid in R. glutinosa, and can provide proposal for utilization of $R$. glutinosa.

\section{Results}

\section{Content of iridoid in R. glutinosa}

The content of iridoid in root increased continuously at growth stage I-III of R. glutinosa, and peaked with $7.55 \%$ at III (Fig. 1 a). After growth stageIII, the content of iridoid was decreased, but was still higher compared to growth stage I . Similar results were found in Fig. 1 (b), where the accumulation of iridoid in leaf was also increased continuously at growth stage I - III of $R$. glutinosa and reached the maximum value at growth stage III (6.52\%). It subsequently began to decrease, but was still higher than that at growth stage I (2.42\%) (Fig. 1, b). Thus, the accumulation of iridoid appeared in a similar trend in root and leaf during the growth and development of $R$. glutinosa, and the content of iridoid in root or leaf was the highest at growth stage III.

Compared with root, the content of iridoid in leaf of $R$. glutinosa was higher at growth stage II, while it was lower at growth stage III - IV $(\mathrm{P}<0.01)$. There was no significant difference between growth stages of $\mathrm{I}$ and $\mathrm{V}$, so the accumulation of iridoid is also important in leaf, especially at growth stage II of $R$. glutinosa, indicating that leaf of $R$. glutinosa could be utilized to alleviate shortage of resources.

\section{Expression of DXR in R. glutinosa}

After growth stage I, the expression of DXR2 was decreased continuously in root, stem or leaf (Fig. 2, a), and its expression peaked at level 1.0, 1.254 or 4.075 , respectively. At the same growth stage of $R$. glutinosa, the expression of DXR2 in leaf was higher as compared to that in root or stem $(P<0.01)$.

As shown in Fig. 2 (b), the expression of DXR4 presented an abrupt increase in root, stem and leaf at growth stage II, subsequently increased tardily, but was still lower, compared with growth stage II . Furthermore, the expression of DXR4 was significantly different in root, stem and leaf at growth stage II, as the following trends: stem $>$ root $>$ leaf $(P<0.01)$, but was higher in leaf at growth stages IV or $V(P<0.01)$. In addition, the expression of DXR4 in root, stem or leaf was respectively higher than that of DXR2 during the growth and development of $R$. glutinosa $(\mathrm{P}<0.01)$.

\section{Expression of GPPS in R. glutinosa}

As shown in Fig. 3, the expression of GPPS3 was higher in root and stem after growth stage I, and peaked at growth stage II -III (Fig. 3a). However, the expression of GPPS3 in leaf reached the peak value at growth stage IV, and was higher than that in root and stem during the growth and development of $R$. glutinosa, which was especially significant at growth stage I , II, or IV $(\mathrm{P}<0.01)$. Furthermore, at growth stage II -III of $R$. glutinosa, the expression of GPPS3 in stem was significantly higher than that in root $(P<0.01)$.
Compared with the expression of GPPS3, similar result was found in GPPS1 (Fig. 3b). The expression of GPPS1 was especially higher in leaf than that in root and stem at growth stages I, II and IV $(\mathrm{P}<0.01)$, but was similar in root and stem during the growth and development of R. glutinosa (Fig. 3b). In addition, the expression of GPPS3 and GPPS1 in R. glutinosa had the similar expression pattern, and there was no difference between them, indicating that GPPS1 and GPPS3 might be one gene, which could encode geranyl pyrophosphate synthase.

\section{Expression of $\mathrm{G1OH}$ in R. glutinosa}

In this study, the expression tendency of $\mathrm{G} 10 \mathrm{H} 1$ and $\mathrm{G} 10 \mathrm{H} 2$ showed similar phenomenon during the growth and development of $R$. glutinosa (Fig. 4). It reached the maximum value in root, stem and leaf at growth stage II, and the minimum value appeared at growth stage $V$.

However, the expression of $\mathrm{G1OH} 1$ and $\mathrm{G1OH} 2$ in root, stem and leaf was different (Fig. 4), at growth stage I. The expression of $\mathrm{G} 10 \mathrm{H} 1$ in leaf and stem was similar, and was significantly higher than that in root (Fig. 4a), yet the significant difference was found among expressions of $\mathrm{G} 1 \mathrm{OH} 2$ in various tissues (Fig. 4b), stem> leaf $>$ root $(P<0.01)$. Furthermore, at growth stage II, the expression of $\mathrm{G} 10 \mathrm{H} 1$ and $\mathrm{G} 1 \mathrm{OH} 2$ both showed the following: leaf $>$ stem $>\operatorname{root}(\mathrm{P}<0.01)$, and was also higher in leaf at growth stage III-IV of $R$. glutinosa $(\mathrm{P}<0.01)$. In addition, the expression of $\mathrm{G} 10 \mathrm{H} 2$ was higher compared to that of $\mathrm{G} 1 \mathrm{OH} 1$ (Fig. 4), conjecturing that $\mathrm{G} 1 \mathrm{OH} 1$ and $\mathrm{G} 1 \mathrm{OH} 2$ might be two different genes encoding geraniol 10-hydroxylase although their expression patterns were consistent.

\section{Expression of $10 \mathrm{HGO}$ in R. glutinosa}

The expression of $10 \mathrm{HGO} 33$ was different in root, stem and leaf of $R$. glutinosa (Fig. 5, a), during the growth and development of $R$. glutinosa. The expression of $10 \mathrm{HGO} 33$ in root had a downward trend, and peaked at level 1.067 in growth stage I $(\mathrm{P}<0.01)$. However, it was up-regulated in stem and reached the maximum value at growth stage $V$. The expression of $10 \mathrm{HGO} 33$ in leaf was also up-regulated, and peaked at level 2.0 in growth stage II $(P<0.01)$, then decreased and was still higher than that at growth stage I .

As shown in Fig. 5b, the expression tendency of $10 \mathrm{HGO6}$ appeared different in root, stem and leaf during the growth and development of R. glutinosa. The expression of $10 H G O 6$ in root and stem both reached maximum value at growth stage II yet was the highest in leaf at growth stage IV. At the same growth stage of $R$. glutinosa, the expression of $10 \mathrm{HGO}$ in various tissues appeared significantly different except for growth stage II-III. For example, the expression of $10 \mathrm{HGO6}$ at growth stage IV-V was significantly higher in leaf and root than that in stem, especially in leaf $(P<0.01)$. Further analysis found that the expression of $10 \mathrm{HGO} 33$ and $10 \mathrm{HGO} 6$ was different in R. glutinosa (Fig. 5), indicating that $10 \mathrm{HGO} 33$ and $10 H G O 6$ may belong to two different genes encoding 10-hydroxygeraniol oxidoreductase. 
Table 1. The primers used in qRT-PCR.

\begin{tabular}{ll}
\hline Primer & Sequence of primer $\left.\left(5^{\prime}-3\right)^{\prime}\right)$ \\
\hline DXR2-F & TCCACTGTAATCTTCTTTCCATA \\
DXR2-R & TCTCAGGCGGATACTTTTGACT \\
DXR4-R & ATTTGGCTTACGCTGCTGG \\
GPPS1-F & TTCTGCTCGGTGCTTATCG \\
GPPS1-R & TCGGGCAACAGGCTCAT \\
GPPS3-F & GAACAGTGGGTCGGAATCTTT \\
GPPS3-R & GATACAAGGAGGGGAATCGG \\
G10H1-F & CCACAACTTTAGCCAAGAGGG \\
G10H1-R & TCCGTCGTCTGGCTTCCG \\
G10H2-F & TTTCCTACACCTGAGATGCTGATT \\
G10H2-R & GTAATGCTTGGTTCGCTTTTG \\
$10 H G O 6-\mathrm{F}$ & GGAGTGGTTGAGCCTTCTGC \\
$10 H G O 6-\mathrm{R}$ & TTCCAGATTCGCCGTAAGC \\
$10 H G O 33-\mathrm{F}$ & CACAGGAGATGATTGATTTGC \\
$10 H G O 33-R$ & GCCTGGGCACGAGATTG \\
TIP41-F & TGAGTATTTGTTGGGGCAGTA \\
TIP41-R & TGGCTCAGAGTTGATGGAGTGCT \\
\hline
\end{tabular}
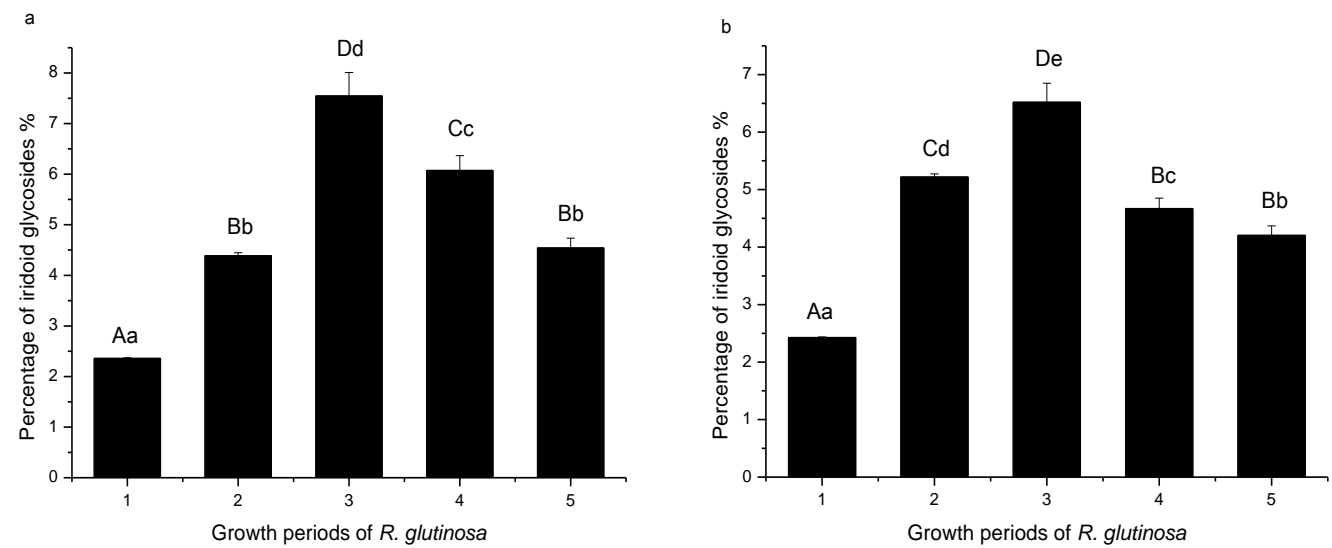

Fig 1. The content of iridoid in R. glutinosa (a) The content of iridoid in root of R. glutinosa; (b) The content of iridoid in leaf of $R$. glutinosa. 1, 2, 3, 4 or 5, respectively, represented plants at growth stage of I , II, III, IV and V in $R$. glutinosa. The lower cases and capital letters represented significant differences $(P<0.05)$ or extremely significant difference $(P<0.01)$, respectively.
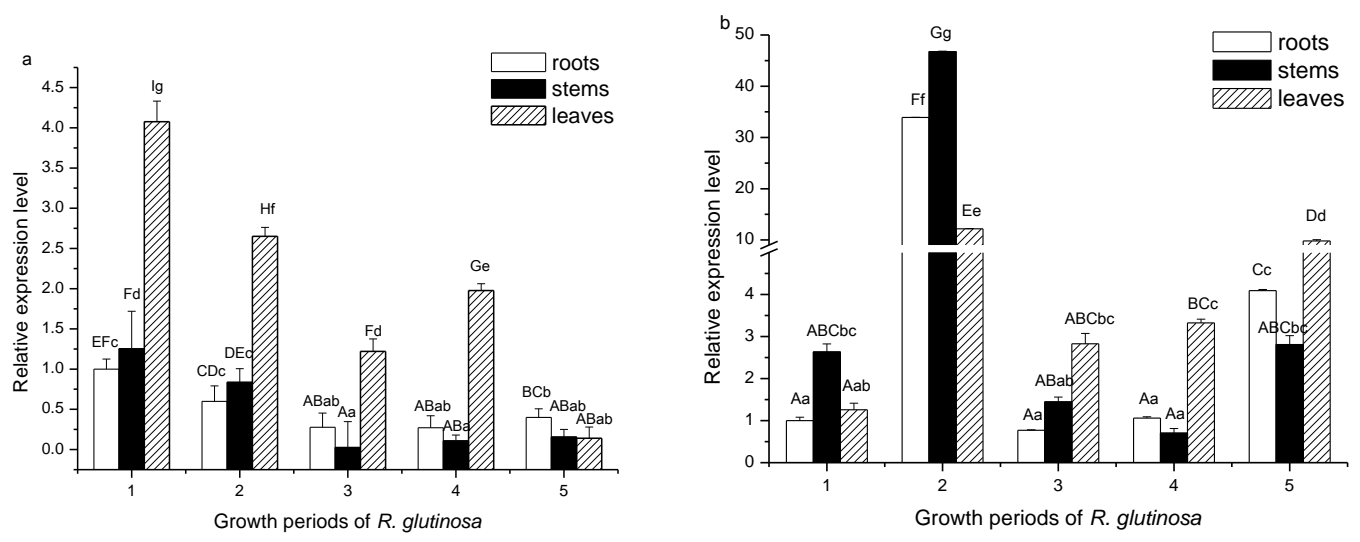

Fig 2. The expression of $D X R$ in $R$. glutinosa (a) and (b) represented the expression of $D X R 2$ or $D X R 4$, respectively. The numbers 1,2 , 3,4 or 5 respectively represented plants at I, II, III, IV and V growth stage of $R$. glutinosa. The lower cases and capital letters represented significant differences $(P<0.05)$ or extremely significant difference $(P<0.01)$, respectively. 

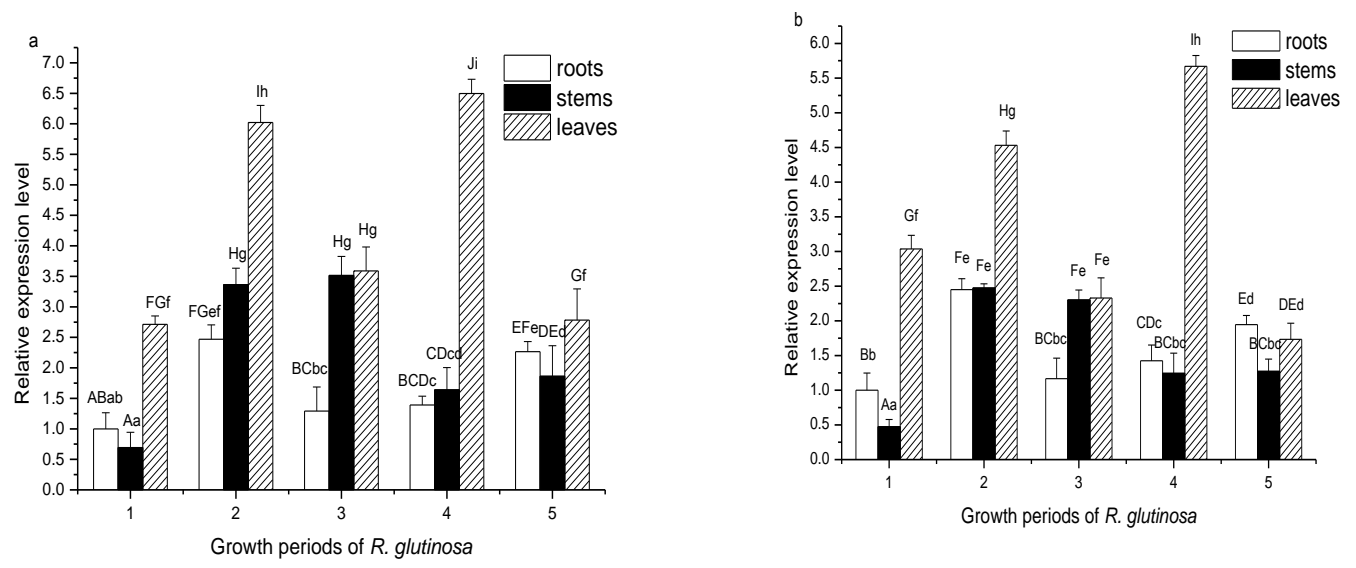

Fig 3. The expression of GPPS in R. glutinosa (a) and (b) represented the expression of GPPS3 and GPPS1 in R. glutinosa, respectively. The numbers $1,2,3,4$ or 5 respectively represented plants at I, II, III, IV and V growth stages. The lower cases and capital letters represented significant differences $(P<0.05)$ or extremely significant difference $(P<0.01)$, respectively.
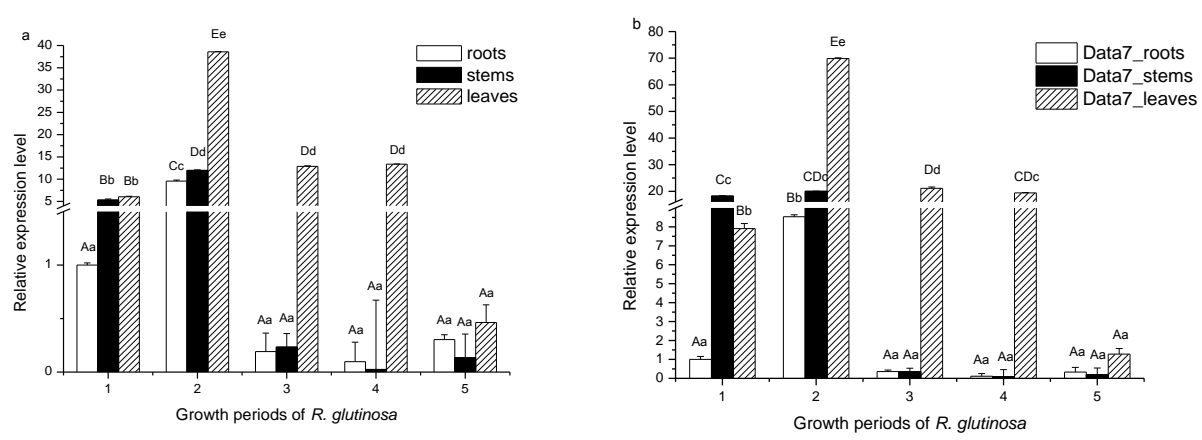

Fig 4. The expression of $\mathrm{G} 10 \mathrm{H}$ in R. glutinosa (a) and (b) represented the expression of $\mathrm{G} 10 \mathrm{H} 1$ and $\mathrm{G} 10 \mathrm{H} 2$ in $R$. glutinosa, respectively. The numbers 1, 2, 3, 4 or 5 represented plants at I , II, III, IV and V growth stage of $R$. glutinosa, respectively. The lower cases and capital letters represented significant differences $(P<0.05)$ or extremely significant difference $(P<0.01)$, respectively.
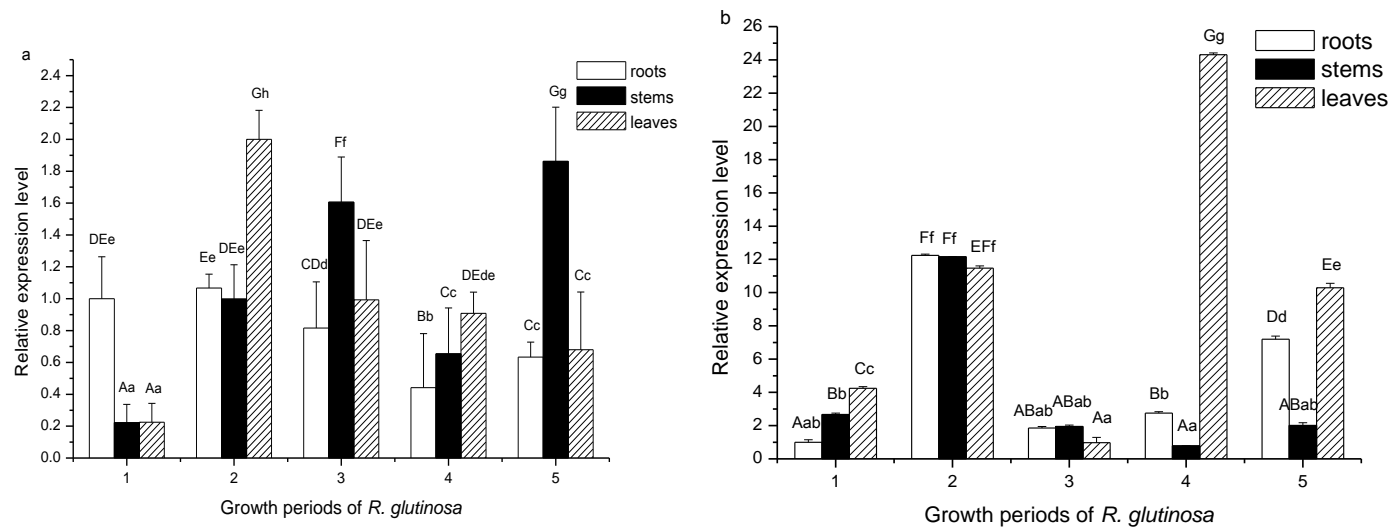

Fig 5. The expression of $10 \mathrm{HGO}$ in R. glutinosa (a) and (b) represented the expression of $10 \mathrm{HGO} 33$ and $10 \mathrm{HGO6}$, respectively. The numbers 1, 2, 3, 4 or 5 represented plants in I , II, III, IV and V growth stage of $R$. glutinosa, respectively. The lower cases and capital letters represented significant differences $(P<0.05)$ or extremely significant difference $(P<0.01)$, respectively. 


\section{Discussion}

It is well known that terpenoid is one important kind of secondary metabolites, and distributes widely in plant (Peng et al., 2002). Iridoid is one kind of terpenoid, exhibits antimicrobial properties and is important in defensive response to bacteria and fungus (Wang et al., 2009). However, the distribution of secondary metabolite is various among species, grow stages and tissues of plants (Nu et al., 2012). In this study, the content of iridoid in $R$. glutinosa increased continuously during the first three growth stages, and reached the maximum value in root and leaf at growth stage III. The similar results were also found in other researches (Ji et al., 2014). In addition, the content of iridoid in leaf of $R$. glutinosa was not lower compared with that in root. Therefore, the leaf of $R$. glutinosa may become one important source of iridoid.

The synthetic pathway of iridoid is mainly MEP, in which it serves as the precursor for many substances, such as IPP, DMAPP, vitamin B1, and so on (Fitzpatrick et al., 2007). Some studies showed that DXR would play a critical role in directing intermediate flux into IPP and DMAPP synthetic pathway (Xing et al., 2010). The GPPS is responsible for enzymatic formation of GPP towards most of monoterpenoid and diterpenoid in plastid (Nagegowda, 2010; Schmidt et al., 2010; Xi et al., 2016). The $\mathrm{G} 10 \mathrm{H}$ is a rate-limiting enzyme for the synthesis of terpenoid (Dagnino et al., 1995; Cui et al., 2015; Kai et al., 2015), and 10HGO could take part in the synthesis of ring-opening iridoid (Ji, 2014). In this study, the expression of two unigenes for DXR, GPPS, G10H and $10 \mathrm{HGO}$ were analyzed in $R$. glutinosa. We found that DXR2 and DXR4 had different expression. The expression of $10 \mathrm{HGO} 6$ and $10 \mathrm{HGO} 33$ was also different, although $\mathrm{G} 1 O H 1$ and $\mathrm{G} 10 \mathrm{H} 2$ had the same expression pattern, their expression level were different. However, the expression of GPPS3 and GPPS1 in R. glutinosa was similar, inferring that GPPS3 and GPPS1 might be two parts of GPPS, which was consistent with the previous research (Ji, 2014). Thus, two unigenes for DXR, G10H and $10 \mathrm{HGO}$ might be both differently regulated during the growth and development of R. glutinosa.

Further analysis showed that expression patterns or levels of DXR, GPPS, G10H, 10HGO were different during the growth and development of $R$. glutinosa, where all appeared tissue specificity, and their expression was relative to the accumulation of iridoid in $R$. glutinosa. This indicates that these enzymes might take part in the synthesis of iridoid in $R$. glutinosa, but could be differently regulated during the growth and development of $R$. glutinosa, which was also found in other plants (Zhu et al., 2016). Given that many steps and enzymes are involved in the synthesis of iridoid in plant (Liu et al., 2004), its metabolism regulation is complex in plant. Therefore, this research provided evidence for synthesis and metabolism regulation of iridoid in $R$. glutinosa. However, the regulatory network of iridoid synthesis is very complicated and need to be further studied in R. glutinosa.

\section{Materials and Methods}

\section{Plant materials}

R. glutinosa Wen 85-5 was used to be experimental material in this study, and its root tubers were kindly provided by Agricultural Research Institute of Wenxian County, Henan, China. Root tubers of $R$. glutinosa were grown in test field,
Wenxian County, Jiaozuo City, Henan, China. Type of planting soil was loam, planting density was $40 \times 40 \mathrm{~cm}$, and $R$. glutinosa was managed by conventional field management. In this study, $R$. glutinosa was analyzed at five different growth stages (Duan et al., 2018), such as growth stage I (seedling root is not fleshy), II (plant root is fleshy and cylindrical), III (plant root is expansive in the middle), IV (plant root appears late expansion), V (plant root is spindle-shaped). Root, stem and leaf of $R$. glutinosa at each growth stage were frozen in liquid nitrogen and stored at $-80^{\circ} \mathrm{C}$ for RNA preparation, or were baked in Electro-Thermostatic Blast Oven at $50^{\circ} \mathrm{C}$ for $48 \mathrm{~h}$ and then crushed into powder.

\section{Determination of iridoid}

Extraction of iridoid from leaf and root of $R$. glutinosa was performed as following: $1.0 \mathrm{~g}$ power of leaf or root was added into $100 \mathrm{~mL}$ conical flask with $10 \mathrm{~mL} 70 \%$ alcohol. Then, it was mixed and put into ultrasonic cleaner for $45 \mathrm{~min}$. After the mixture was filtered, the filtrate was added into $50 \mathrm{~mL}$ volumetric flask with $70 \%$ alcohol, then $2.5 \mathrm{~mL}$ mixture was drawn into another $50 \mathrm{~mL}$ volumetric flask with $70 \%$ alcohol and used to be reaction liquid. The content of iridoid was measured at $463 \mathrm{~nm}$ with ultraviolet (UV) spectrophotometer according to methods by Zeng (Zeng et al., 2010).

In addition, $5.5 \mathrm{mg}$ catalpol reference substance was dissolved with $70 \%$ alcohol to make the concentration of catalpol solution of $0.22 \mathrm{mg} / \mathrm{L}$, then $1.5,2.0,2.5,3.0,3.5$ or $4.0 \mathrm{~mL}$ solution was drawn into $10 \mathrm{~mL}$ volumetric flask and was constant with $70 \%$ alcohol, respectively. The measurement method of catalpol reference substance was same to that of reaction liquid, and the standard curve was in the following, $y=0.0754 * x+0.065, R^{2}=0.998$ ( $y$ : concentration, $x$ : absorbance).

\section{Extraction of total RNA}

Total RNA was extracted from root, stem and leaf of $R$. glutinosa with RNAiso Plus (TaKaRa, Japan) according to the instructions. Furthermore, in this study, DNase/RNase-free treatment and phenol-chloroform extraction were used to remove DNase, and RNA was dissolved in RNase-free $\mathrm{dH}_{2} \mathrm{O}$. The integrity of total RNA was verified by $1.0 \%$ agarose gel, the yield of total RNA was determined at $260 \mathrm{~nm}$ with UV spectrophotometer, and the purity of total RNA was checked by determining A260/A280 ratio.

\section{qRT-PCR}

Expression of key enzymes taking part in the synthesis of iridoid such as DXR, GPPS, G10H and $10 \mathrm{HGO}$ was studied. According to transcriptome sequencing results of $R$. glutinosa, two unigenes for each key enzyme were chosen as DXR2, DXR4, GPPS1, GPPS3, 1OHGO6, 10HGO33, G1OH1 and G1OH2, respectively (Table 1 ). In this study, the internal reference gene was TIP41, all primers were synthesized by Yingjie Ji Trade Co., Ltd. (Shanghai, China) and their sequences were listed in Table 1.

The qRT-PCR was conducted by LightCycler 96 Real-time PCR reaction, and CDNA used in GRT-PCR was synthesized by PrimeScript RT reagent Kit with gDNA Eraser (Perfect Real Time: TaKaRa, Japan). The total volume of qRT-PCR reaction system was $20 \mu \mathrm{l}$, composed of $10 \mu \mathrm{l} 2 \times$ SYBR Premix Ex Taq II (TliRNaseH Plus) (TaKaRa, Japan), $0.5 \mu$ l each primer $(10 \mu \mathrm{M}), 2$ 
$\mu \mathrm{l}$ of diluted cDNA mix and $7 \mu \mathrm{ld} \mathrm{H}_{2} \mathrm{O}$. The reaction procedure of qRT-PCR was $95^{\circ} \mathrm{C}$ for 30 s, followed by 40 cycles of $95^{\circ} \mathrm{C}$ for $5 \mathrm{~s}, 60^{\circ} \mathrm{C}$ for $30 \mathrm{~s}$, then for melting carve stage. In addition, the relative expression level of gene was normalized and analyzed by the comparative Ct $\left(2^{-\Delta \Delta c t}\right)$ method (Livak and Schmittgen, 2001).

\section{Statistical analysis of data}

Statistical analysis of data in this study was performed as the following: content of iridoid and expression of genes were tested by significance level, ANOVA (analysis of variance) and multiple comparisons of Duncan's multiple range. Content of iridoid and expression of genes were calculated and analyzed by Excel, and all histograms were drawn by Origin.

\section{Conclusion}

In this study, the content of iridoid was increased continuously during the growth and development of $R$. glutinosa. It reached the maximum value at growth stage III, and the content of iridoid in leaf was not lower as compared with that in root. Furthermore, expression pattern or level of DXR, GPPS, G1OH, $10 H G O$ was different in various tissues and stages of $R$. glutinosa, and their expression was related to the accumulation of iridoid in $R$. glutinosa. Thus, this research would help to study metabolic pathway of iridoid in $R$. glutinosa, and can provide proposal for utilization of $R$. glutinosa.

\section{Acknowledgments}

This research was supported by Natural Science Foundation of China (Grant No. 31870312, 31500262), and Project of Science and Technology Innovative Talent Fund in Henan University (Grant No. 17HASTIT034).

\section{References}

Ashour M, Wink M, Gershenzon J (2010) Biochemistry of Terpenoids: Monoterpenes, Sesquiterpenes and Diterpenes. In: Wink $M$, editor. Biochemistry of plant secondary metabolism. Chap. 5. Oxford: Wiley-Blackwell, 258-303.

Cui L, Ni X, Ji Q, Teng X, Yang Y, Wu C, Zekria D, Zhang D, Kai G (2015) Co-overexpression of geraniol-10-hydroxylase and strictosidine synthase improves anti-cancer drug camptothecin accumulation in Ophiorrhiza pumila. Sci Rep. 5: 8227.

Dagnino D, Schripsema J, Verpoorte R (1995) Terpenoid indole alkaloid biosynthesis and enzyme activities in two cell lines of Tabernaemontana divaricata. Phytochemistry. 39: 341-349.

Duan HY, Li JJ, Wang HN, Chen FF, Ding WK, Zhu YQ, Zheng X, Zeng YP, Zhou YQ (2018) Effects of DNA methylation on growth and development of Rehmannia glutinosa. Int J Agric Biol. 20: 2161-2168.

Fitzpatrick T, Amrhein N, Kappes B, Macheroux P (2007) Two independent routs of de novo Vitamin B6 biosynthesis: not that different at all. Biochem J. 407: 1-13.

Ji X, Sun P, Qi J, Liao D, Li XE (2014) Study on distribution and dynamic accumulation of catalpol and total iridoid in fresh Rehmannia glutinosa. China J Chin Mater Medica. 3: 466-470.
Ji XQ (2014) Study on the content changes of catalpol in Rehmannia glutinosa and gene expression of key enzymes in terpene synthesis. Master's thesis of Peking Union Medical College.

Kai G, Wu C, Gen L, Zhang L, Cui L, Ni, X (2015) Biosynthesis and biotechnological production of anti-cancer drug camptothecin. Phytochem Rev. 14: 525-539.

Kang KH, Kim CH (2011) Inhibitory effect of Rehmannia Glutinosa pharmacopuncture solution on $\beta$-hexosaminidase release and cytokine production via immunoglobulin receptor signaling in RBL-2H3 cells. J Acupuncture and Meridian Studies. 4: 269.

Laule O, Fürholz A, Chang HS, Zhu T, Wang X, Heifetz PB, Gruissem W, Lange M (2003) Crosstalk between cytosolic and plastidial pathways of isoprenoid biosynthesis in Arabidopsis thaliana. P Nati Acad Sci. 100: 6866-6871.

Li H, Yang SQ, Wang H, Tian J, Gao WY (2010) Biosynthesis of the iridoid glucoside, lamalbid, in Lamium barbatum. Phytochem. 71: 1690-1694.

Liu J, Ling JY, Xie T (2004) Research progress of iridoid compounds. Strait Pharm. 16: 14-19.

Luo YM, Liu AH, Li Q (2003) Biosynthetic pathways and key enzymes of plant terpenoids research progress. J Jiangxi Coll TCM. 45-51.

Nagegowda DA (2010) The small subunit of geranyl diphosphate synthase: a tool to improve aroma and flavour by metabolic engineering. J Biosci. 35:167-169.

Newman JD, Chappell J (2008) Isoprenoid biosynthesis in plants: carbon partitioning within the cytoplasmic pathway. Crit Rev Biochem Mol Biol. 34: 95-106.

$\mathrm{Nu}$ Y, Zhou XD, Lou ZH, Xiao XY (2012) Review of plant secondary metabolites and the factors that influence its accumulation. Jiangxi For Sci Technol. 3: 54-60.

Peng SL, Nan P, Zhong Y (2002) Terpenoids in higher plants and their roles in ecosystems. Chin J Ecol. 21: 33-38.

Schmidt A, Wächtler B, Temp $U$, Krekling T, Séguin A, Gershenzon J (2010) A bifunctional geranyl and geranylgeranyl diphosphate synthase is involved in terpene oleoresin formation in Picea abies. Plant Physiol. 152: 639-655.

Sung PH, Huang FC, Do YY, Huang PL (2011) Functional expression of geraniol 10-hydroxylase reveals its dual function in the biosynthesis of terpenoid and phenylpropanoid. J Agri Food Chem. 9: 4637-4643.

Thamm AMK, Qu Y, Luca VD (2016) Discovery and metabolic engineering of iridoid/secoiridoid and monoterpenoid indole alkaloid biosynthesis. Phytochem Rev. 15: 339-361.

Verma P, Mathur AK, Srivastava A, Mathur A (2012) Emerging trends in research on spatial and temporal organization of terpenoid indole alkaloid pathway in Catharanthus roseus: a literature update. Protoplasma. 2: 255-268.

Wang CT, Liu H, Gao XS, Zhang HX (2010) Overexpression of $\mathrm{G} 10 \mathrm{H}$ and ORCA3 in the hairy roots of Catharanthus roseus improves catharanthine production. Plant Cell Rep. 29: 887-894.

Wang T, Gui M, Liu H, Zhao H, Xu L, Zha M, Li JY (2009) Secretion of catalpol from Rehmannia glutinosa. Acta Physiol Plant. 32: 141-144.

Xi J, Rossi L, Lin X, Xie DY (2016) Overexpression of a synthetic insect-plant geranyl pyrophosphate synthase gene in Camelina sativa. Planta. 244: 215-230. 
Xing S, Miao J, Li S, Qin G, Tang S, Li H, Gu H, Qu L (2010) Disruption of the 1-deoxy-D-xylulose-5-phosphate reductoisomerase (DXR) gene results in albino, dwarf and defects in trichome initiation and stomata closure in Arabidopsis. Cell Res. 20: 688-700.

Yang L, Yang C, Li C, Zhao Q, Liu L, Fang X, Chen X (2016) Recent advances in biosynthesis of bioactive compounds in traditional Chinese medicinal plants. Sci Bull. 61: 3-17.

Yu X, Wang HT, Feng MY, Liang CY, Li WL (2013) Cloning and expression analysis of gene GPPS in mint. Acta Agric Jiangxi. 7: 25-29.

Zeng LF, Liu XL, Wang HC (2010) Spectrophotometric determination of total iridoid glycosides in Rehmannia. Chin J Anal Lab. 210-212.

Zhang Y, Feng W, Zheng X, Cao Y, Lv Y, Chen H, Kuang H (2013) Three new ursane-type triterpenes from the leaves of Rehmannia glutinosa. Fitoterapia. 89: 15-19.
Zhou YQ, Yang K, Zhang DD, Duan HY, Liu YK, Guo MM (2018) Metabolite accumulation and metabolic network in developing roots of Rehmannia glutinosa reveals its root developmental mechanism and quality. Sci Rep. 8: 14127 DOI:10.1038/s41598-018-32447-6.

Zhu JL, Zhao L, Dong CM, Zheng XK, Feng WS (2016) Analysis of genes involved in the synthesis and modification of iridoid of Rehmannia glutinosa. Modern Food Science and Technology. 10: 84-89. 